\title{
Exome sequencing reveals frequent deleterious germline variants in cancer susceptibility genes in women with invasive breast cancer undergoing neoadjuvant chemotherapy
}

\author{
Marissa S. Ellingson ${ }^{1} \cdot$ Steven N. Hart $^{2} \cdot$ Krishna R. Kalari $^{2}$ • Vera Suman ${ }^{2}$ • \\ Kimberly A. Schahl ${ }^{1} \cdot$ Travis J. Dockter $^{2} \cdot$ Sara J. Felten $^{2} \cdot$ Jason P. Sinnwell $^{2}$. \\ Kevin J. Thompson ${ }^{2}$ - Xiaojia Tang ${ }^{2}$ Peter T. Vedell ${ }^{2}$ Poulami Barman ${ }^{2}$. \\ Hugues Sicotte $^{2}$ - Jeanette E. Eckel-Passow ${ }^{2}$ - Donald W. Northfelt ${ }^{3}$. \\ Richard J. Gray ${ }^{4}$ - Sarah A. McLaughlin ${ }^{5}$ - Alvaro Moreno-Aspitia ${ }^{6}$. \\ James N. Ingle ${ }^{13}$ • Ann M. Moyer ${ }^{7}$ - Daniel W. Visscher ${ }^{7}$ Katie Jones $^{8}$. \\ Amy Conners ${ }^{8} \cdot$ Michelle McDonough $^{9} \cdot$ Eric D. Wieben ${ }^{10} \cdot$ Liewei Wang $^{11}$. \\ Richard Weinshilboum ${ }^{1,11} \cdot$ Judy C. Boughey ${ }^{12} \cdot$ Matthew P. Goetz $^{12,13}$
}

Received: 8 August 2015/Accepted: 10 August 2015/Published online: 22 August 2015

(C) The Author(s) 2015

\begin{abstract}
When sequencing blood and tumor samples to identify targetable somatic variants for cancer therapy, clinically relevant germline variants may be uncovered. We evaluated the prevalence of deleterious germline variants in cancer susceptibility genes in women with breast cancer referred for neoadjuvant chemotherapy and returned clinically actionable results to patients. Exome sequencing was performed on blood samples from women with invasive breast cancer referred for neoadjuvant chemotherapy. Germline variants within 142 hereditary cancer susceptibil-
\end{abstract}

Electronic supplementary material The online version of this article (doi:10.1007/s10549-015-3545-6) contains supplementary material, which is available to authorized users.

Judy C. Boughey

Boughey.Judy@mayo.edu

Matthew P. Goetz

Goetz.Matthew@mayo.edu

1 Center for Individualized Medicine, Mayo Clinic, 200 First Street SW, Rochester, MN 55905, USA

2 Department of Health Sciences Research, Mayo Clinic, 200 First Street SW, Rochester, MN 55905, USA

3 Hematology/Oncology, Mayo Clinic, 13400 East Shea Blvd, Scottsdale, AZ 85259, USA

4 Department of Surgery, Mayo Clinic, 13400 East Shea Blvd, Scottsdale, AZ 85259, USA

5 Department of Surgery, Mayo Clinic, 4500 San Pablo Road, Jacksonville, FL 32224, USA

6 Hematology/Oncology, Mayo Clinic, 4500 San Pablo Road, Jacksonville, FL 32224, USA ity genes were filtered and reviewed for pathogenicity. Return of results was offered to patients with deleterious variants in actionable genes if they were not aware of their result through clinical testing. 124 patients were enrolled (median age 51) with the following subtypes: triple negative $(n=43,34.7 \%)$, HER $2+(n=37,29.8 \%)$, luminal B $(n=31,25 \%)$, and luminal A $(n=13,10.5 \%)$. Twentyeight deleterious variants were identified in 26/124 (21.0\%) patients in the following genes: $\operatorname{ATM}(n=3), B L M(n=1)$, BRCA1 $(n=4)$, BRCA2 $(n=8)$, CHEK2 $(n=2), F A N C A$ $(n=1), F A N C I(n=1), F A N C L(n=1), F A N C M(n=1)$, FH $(n=1)$, MLH3 $(n=1)$, MUTYH $(n=2)$, PALB2 $(n=1)$, and $W R N(n=1) .121 / 124(97.6 \%)$ patients

7 Department of Laboratory Medicine and Pathology, Mayo Clinic, 200 First Street SW, Rochester, MN 55905, USA

8 Department of Radiology, Mayo Clinic, 200 First Street SW, Rochester, MN 55905, USA

9 Department of Radiology, Mayo Clinic, 4500 San Pablo Road, Jacksonville, FL 32224, USA

10 Biochemistry, Mayo Clinic, 200 First Street SW, Rochester, MN 55905, USA

11 Department of Molecular Pharmacology \& Experimental Therapeutics, Mayo Clinic, 200 First Street SW, Rochester, MN 55905, USA

12 Department of Surgery, Mayo Clinic, 200 First Street SW, Rochester, MN 55905, USA

13 Medical Oncology, Mayo Clinic, 200 First Street SW, Rochester, MN 55905, USA 
consented to return of research results. Thirteen $(10.5 \%)$ had actionable variants, including four that were returned to patients and led to changes in medical management. Deleterious variants in cancer susceptibility genes are highly prevalent in patients with invasive breast cancer referred for neoadjuvant chemotherapy undergoing exome sequencing. Detection of these variants impacts medical management.

Keywords Breast cancer - Neoadjuvant chemotherapy · High-risk breast cancer - Return of results · Exome sequencing - Germline mutation/pathogenic germline variant

\section{Introduction}

Advances in genomic sequencing have resulted in opportunities to individualize patient care. The advent of nextgeneration sequencing has allowed for interrogation of the genome at a significantly reduced cost and may provide the opportunity for some cancer patients to pursue genomeguided therapy by identifying targetable somatic variants [18]. In order to determine which variants are unique to the tumor, germline sequence variants are subtracted from the tumor sequence [16]. Through this process, clinically important germline variants may be uncovered [3]. Although these variants are often labeled "incidental findings," research has demonstrated that the identification of deleterious variants causative of hereditary cancer syndromes should be anticipated in individuals undergoing next-generation sequencing tests $[2,5,7,10]$. The identification of such variants can have a significant impact on the clinical management of a patient, including prophylactic surgeries, surveillance protocols, tailored screening, or change in therapy (e.g., chemoprevention).

The Breast Cancer Genome-Guided Therapy Study (BEAUTY) is a clinical study for patients with newly diagnosed breast cancer referred for neoadjuvant chemotherapy. The primary goal of BEAUTY is to identify novel somatic mutations associated with response to neoadjuvant chemotherapy. An additional goal of the study, which is presented here, is to determine the prevalence of deleterious germline variants in cancer susceptibility genes in these patients. Additionally, we assessed whether patients would desire to receive their germline research results and developed a procedure for return of clinically actionable results.

\section{Methods}

\section{Participant eligibility and accrual}

Patients were enrolled in BEAUTY (NCT02022202) from March 5, 2012, to May 1, 2014. Inclusion criteria were patients age $\geq 18$ years with newly diagnosed stage I-III breast cancer who were recommended for NAC.

\section{Sample preparation, whole exome sequencing, and bioinformatics analyses}

Methodology is described in the Online Resources.

\section{Gene selection}

A list of 142 genes associated with hereditary cancers (Online Resources, Table I) was developed by reviewing clinically available hereditary cancer gene panels, the Concise Handbook of Familial Cancer Susceptibility Syndromes, Online Mendelian Inheritance in Man (OMIM), and published literature [15, 17, 24]. Genes were divided into two tiers for analysis; tier one included genes associated with hereditary breast cancer while tier two included genes associated with other hereditary cancers.

\section{Variant filtering and classification}

Germline variants were filtered to identify missense, nonsense, frameshift, and splice-site variants within 142 hereditary cancer susceptibility genes. We also filtered for all intronic variants captured by exome sequencing in this list of genes that were previously reported as deleterious or pathogenic in the Human Gene Mutation Database (HGMD) or ClinVar [13, 25]. Variants were classified by a Certified Genetic Counselor according to the 2007 American College of Medical Genetics (ACMG) recommendations as either deleterious (category 1), likely deleterious (category 2), variant of uncertain significance (category 3), likely benign (category 4), or benign (category 5) [21]. Variant classification was determined based on reported minor allele frequencies from the Exome Variant Server, 1000 Genomes, and dbSNP; predicted protein impact; in silico models; review of databases such as HGMD, ClinVar, and locus specific databases; and review of published literature $[1,8,13,22,25,26]$.

After classifying variants from the first 91 patients, filtering strategies were designed that reduced the total number of variants for review. In the remaining patients, all tier two variants were filtered to include only those with a minor allele frequency of less than one percent that were either classified as a "disease causing mutation" in HGMD or predicted to be protein truncating [25]. Tier one variants were not filtered in this manner given our desire to maximize sensitivity and the greater likelihood of identifying clinically actionable variants in breast cancer-associated genes.

As an internal validation, we randomly selected approximately $10 \%$ of all variants, making sure to sample all deleterious and likely deleterious variants (hereafter, 
collectively termed deleterious). These variants were then independently classified by a second Certified Genetic Counselor. In scenarios where variant classifications were discrepant between the reviewers, the reviewers met to discuss the discrepancy and to reach a consensus about the final classification.

\section{Return of results}

Patients were invited to consent to the return of germline research results. Patients who consented to the return of results were informed that they would be contacted by a genetic counselor if a clinically actionable deleterious result that the patient was unaware of was identified. After identification of a clinically actionable deleterious variant, a study coordinator contacted eligible patients by telephone to offer an appointment with a genetic counselor. Risks, benefits, and limitations of receiving results were discussed during the genetic counseling appointment. If the patient was interested in receiving results, a second appointment was scheduled for results disclosure. Appointments occurred in-person or by telephone. It was highly recommended that patients proceed with confirmatory testing in a Clinical Laboratory Improvement Amendments (CLIA)certified laboratory prior to making any medical management decisions based on the research results.

From the list of 142 cancer susceptibility genes (Online Resources, Table I), a subset of 39 genes were chosen for inclusion in the return of results protocol as they were determined to be "clinically actionable" based upon having existing medical management guidelines (National Comprehensive Cancer Network or other guidelines in published literature available on PubMed). Return of results was not performed for childhood-onset conditions, autosomal recessive conditions (as it was not possible to determine whether two variants identified within a gene were in $c i s$ or in trans), or carrier status for autosomal recessive conditions.

\section{Ethics}

This study was approved by the Mayo Clinic Institutional Review Board. All patients were required to provide written informed consent before participation, and patients were consented to the use of blood and tumor samples for whole exome sequencing as well as return of results

\section{Results}

\section{Patient characteristics}

The patient and disease characteristics are provided in Table 1. The median age was 51 (range 21-73) and most women had high-risk disease based upon the clinical T-stage [median tumor size $5 \mathrm{~cm}$ (1.1-9.9)], nodal status (56.4\% node positive), and clinical molecular subtype [89.5\% with luminal B, HER2+, or triple negative breast cancer (TNBC)].

\section{Clinical genetic test results}

Review of medical records showed that of the 124 patients enrolled, $81(65.3 \%)$ discussed the option of genetic testing with a clinical provider independent of the study, and 66/81 (81.5\%) underwent clinical genetic testing, including: single site BRCAl $(n=2)$, BRCA1/BRCA2 sequencing $(n=7), B R C A 1 / B R C A 2$ sequencing and deletion/duplication analysis $(n=45), B R C A 1 / B R C A 2$ and TP53 sequencing and deletion/duplication analysis $(n=2)$, a hereditary breast cancer panel $(n=9)$, and immunohistochemistry screening for Lynch syndrome $(n=1)$ (Online Resources, Table II). Nine patients tested positive for deleterious germline variants, including four in $B R C A 1$ and five in BRCA2.

\section{Whole exome sequencing, variant filtering, and variant classification}

After filtering for missense, nonsense, frameshift, splicesite, and previously reported deleterious/pathogenic intronic variants, 694 unique variants (observed 8214 times) were identified in 111 (78.2\%) of the 142 hereditary cancer susceptibility genes examined. Twenty-eight variants were classified as deleterious or likely deleterious, and were present in 26 of the $124(21.0 \%)$ patients in the following genes: ATM $(n=3), \operatorname{BLM}(n=1)$, BRCA1 $(n=4), \quad B R C A 2 \quad(n=8), \quad C H E K 2 \quad(n=2), \quad F A N C A$ $(n=1), \quad F A N C I \quad(n=1), \quad F A N C L \quad(n=1), \quad F A N C M$ $(n=1), F H(n=1), \operatorname{MLH3}(n=1), \operatorname{MUTYH}(n=2)$, PALB2 $(n=1)$, and WRN $(n=1)$ (Table 2). Twenty-four of the $124(19.4 \%)$ patients had one deleterious variant while two patients $(1.6 \%)$ had two deleterious variants.

For the internal validation of variant classification, 81 variants were selected, independently reviewed, and classified by a second Certified Genetic Counselor. Twelve (14.8\%) were discrepant between the reviewers, including five deleterious versus likely deleterious, three likely deleterious versus uncertain significance, three uncertain significance versus likely benign, and one likely benign versus benign. The reviewers met to discuss the discrepancies and a consensus classification was reached for all variants.

All nine deleterious variants detected clinically in a CLIA-certified laboratory (four in $B R C A l$ and five in $B R C A 2)$ were identified by our research study procedures. Classification of these nine variants was concordant in seven cases; two BRCA2 variants that were classified as 
Table 1 Patient characteristics according to the presence (yes/ no) of a deleterious or likely deleterious mutation

\begin{tabular}{|c|c|c|c|}
\hline & No $(N=98)$ & Yes $(N=26)$ & Total $(N=124)$ \\
\hline \multicolumn{4}{|l|}{ Age group } \\
\hline$<30$ & $1(1.0 \%)$ & $1(3.8 \%)$ & $2(1.6 \%)$ \\
\hline $30-39$ & $17(17.3 \%)$ & $4(15.4 \%)$ & $21(16.9 \%)$ \\
\hline $40-49$ & $27(27.6 \%)$ & $7(26.9 \%)$ & $34(27.4 \%)$ \\
\hline $50-59$ & $27(27.6 \%)$ & $10(38.5 \%)$ & $37(29.8 \%)$ \\
\hline $60-69$ & $19(19.4 \%)$ & $3(11.5 \%)$ & $22(17.7 \%)$ \\
\hline $70+$ & $7(7.1 \%)$ & $1(3.8 \%)$ & $8(6.5 \%)$ \\
\hline \multicolumn{4}{|l|}{ Race } \\
\hline White & $86(87.8 \%)$ & $24(92.3 \%)$ & $110(88.7 \%)$ \\
\hline Black or African American & $5(5.1 \%)$ & $2(7.7 \%)$ & $7(5.6 \%)$ \\
\hline Asian & $3(3.1 \%)$ & $0(0.0 \%)$ & $3(2.4 \%)$ \\
\hline American Indian or Alaska Native & $1(1.0 \%)$ & $0(0.0 \%)$ & $1(0.8 \%)$ \\
\hline Unknown: patient unsure & $3(3.1 \%)$ & $0(0.0 \%)$ & $3(2.4 \%)$ \\
\hline \multicolumn{4}{|l|}{ Clinical molecular subtype } \\
\hline Luminal A & $9(9.2 \%)$ & $2(7.7 \%)$ & $11(8.9 \%)$ \\
\hline Luminal B & $23(23.5 \%)$ & $8(30.8 \%)$ & $31(25.0 \%)$ \\
\hline Luminal Unknown & $1(1.0 \%)$ & $1(3.8 \%)$ & $2(1.6 \%)$ \\
\hline ER+/HER2+ & $17(17.3 \%)$ & $0(0.0 \%)$ & $17(13.7 \%)$ \\
\hline ER-/HER2+ & $16(16.3 \%)$ & $4(15.4 \%)$ & $20(16.1 \%)$ \\
\hline Triple negative & $32(32.7 \%)$ & $11(42.3 \%)$ & $43(34.7 \%)$ \\
\hline \multicolumn{4}{|l|}{ Clinical T-stage } \\
\hline $\mathrm{T} 1$ & $10(10.2 \%)$ & $2(7.7 \%)$ & $12(9.7 \%)$ \\
\hline $\mathrm{T} 2$ & $41(41.8 \%)$ & $11(42.3 \%)$ & $52(41.9 \%)$ \\
\hline $\mathrm{T} 3$ & $44(44.9 \%)$ & $12(46.2 \%)$ & $56(45.2 \%)$ \\
\hline $\mathrm{T} 4$ & $3(3.1 \%)$ & $1(3.8 \%)$ & $4(3.2 \%)$ \\
\hline \multicolumn{4}{|l|}{ Clinical N-stage } \\
\hline No & $43(43.9 \%)$ & $11(42.3 \%)$ & $54(43.5 \%)$ \\
\hline N1 & $49(50.0 \%)$ & $14(53.8 \%)$ & $63(50.8 \%)$ \\
\hline $\mathrm{N} 2$ & $3(3.1 \%)$ & $1(3.8 \%)$ & $4(3.2 \%)$ \\
\hline N3 & $3(3.1 \%)$ & $0(0.0 \%)$ & $3(2.4 \%)$ \\
\hline
\end{tabular}

pathogenic/deleterious by the clinical laboratory were classified as likely deleterious in our study. Four additional deleterious variants in BRCA2 and $F H$ were detected in our study that had not been tested for clinically.

162 unique variants of uncertain significance (VUS) were found in 57/142 $(40.1 \%)$ genes examined among $103 / 124(83.1 \%)$ patients. The number of VUS per patient ranged from 0 to 6 (median 2.0). The number of unique VUS per gene (excluding genes containing no variants) ranged from 0 to 21 (median 2.0), with ATM containing the highest number (21 unique VUS).

No large deletions or duplications were observed.

\section{Tumor characteristics in patients with deleterious variants}

The approximated clinical subtypes among the 26 patients with one or more deleterious variants included $11(42.3 \%)$ TNBCs, four (15.4\%) HER2+, two (7.7 \%) luminal A, eight $(30.8 \%)$ luminal $\mathrm{B}$, and one $(3.8 \%)$ luminal unknown breast cancer (Table 1). In contrast, the clinical subtypes among the 98 patients without a deleterious variant included 32 (32.7\%) TNBCs, 33 (33.6\%) HER2+, nine (9.2\%) luminal A, 23 (23.5\%) luminal B, and one $(1.0 \%)$ luminal unknown breast cancer.

\section{Return of results}

Nearly all of the patients $(121 / 124 ; 97.6 \%)$ consented to the return of germline research results. Thirteen patients were found to carry a deleterious variant in a cancer susceptibility gene that met the criteria for return of test results (Table 2). Four of these 13 were not already aware of their mutation through clinical testing. Their mutations included one likely deleterious $B R C A 2$ variant, two deleterious $B R C A 2$ variants, and a likely deleterious $F H$ (Fumarate hydratase, causative of Hereditary Leiomyomatosis and Renal Cell Cancer) variant. The presence of each of these 


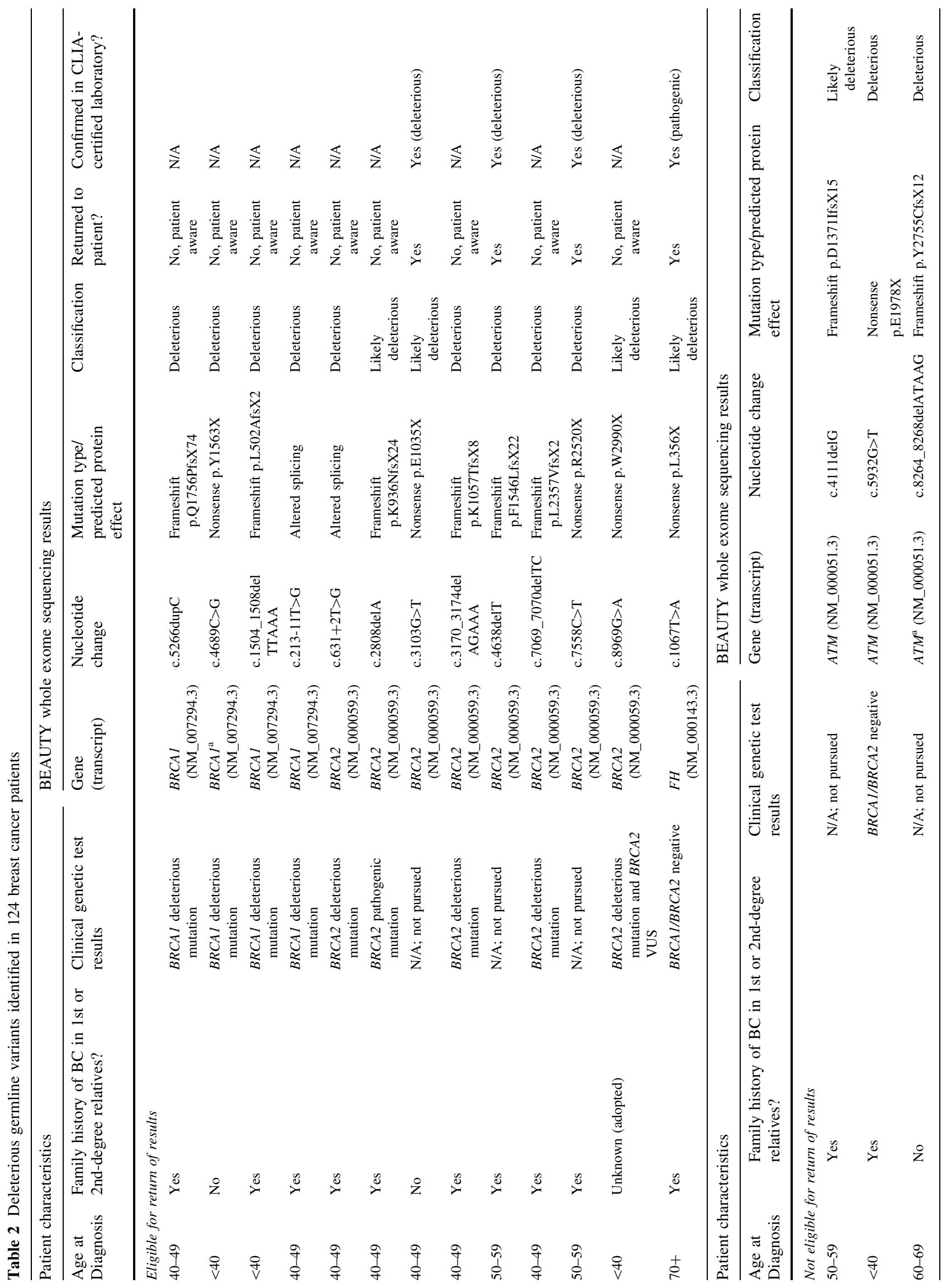




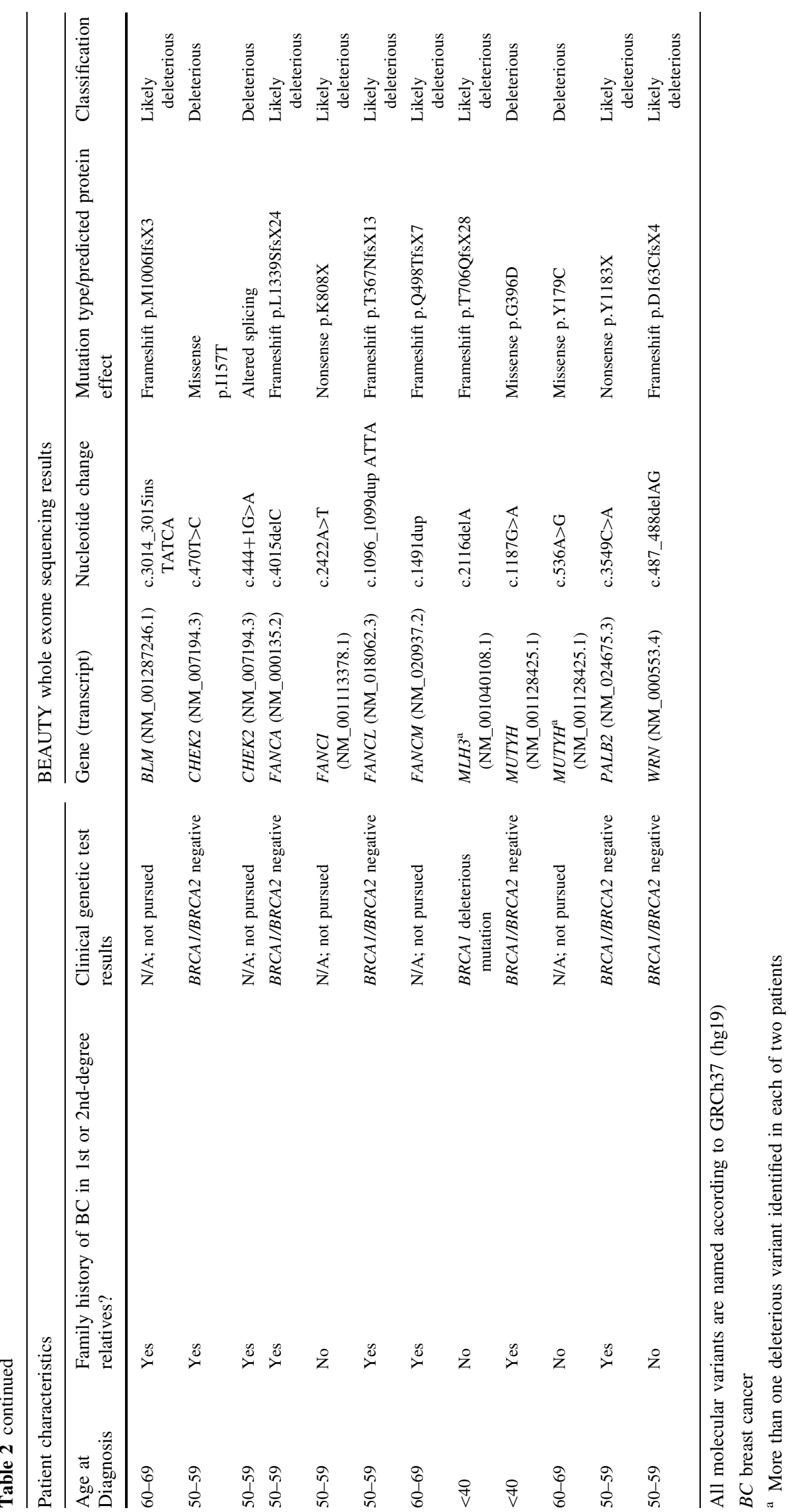


four variants was subsequently confirmed in a CLIA-certified laboratory.

\section{Frequency of deleterious variants in databases}

Of the 28 unique deleterious variants, 16 were classified as "DM" (disease causing mutation) in HGMD and as pathogenic in ClinVar. Two variants were classified as DM in HGMD but were not reported in ClinVar, and two were classified as pathogenic in ClinVar but were not reported in HGMD. One variant was classified as pathogenic in ClinVar and as a "disease-associated polymorphism with additional supporting functional evidence" in HGMD. One variant was classified as "DM?" (likely disease causing but with questionable pathogenicity) in HGMD and not reported in ClinVar. All of the remaining six deleterious variants that were not reported in either of the databases were predicted to be protein truncating (nonsense, frameshift, or splice-site variants). The deleterious variants absent from ClinVar will be submitted for inclusion.

\section{Discussion}

In a prospective study of women with invasive breast cancer recommended to undergo NAC, deleterious germline variants in cancer susceptibility genes were highly prevalent and identified in 26 of $124(21.0 \%)$ patients. These variants were identified in a number of breast cancer susceptibility genes, including ATM, BRCA1, BRCA2, $C H E K 2$, and $P A L B 2$. Furthermore, we identified variants in a variety of other cancer susceptibility genes including BLM, FANCA, FANCI, FANCL, FANCM, FH, MLH3, $M U T Y H$, and $W R N$. Although the latter variants have not been classically associated with breast cancer, evidence implicates a potential role for several of these genes in the pathogenesis of breast cancer including $B L M[6,19,27]$, FANCA [23], FANCM [9], FH [14], MLH3 [4], MUTYH [20], and WRN [28].

Thirteen patients were found to carry deleterious variants in actionable genes (Table 2). While the majority of patients with actionable variants were already aware of their result through clinical testing, we identified four patients with deleterious/likely deleterious variants who were not aware of their results, including three in BRCA2 and one in $F H$. All four variants were confirmed by CLIAcertified laboratories. For these patients, the medical management plans changed significantly including plans for a prophylactic bilateral salpingo-oophorectomy (BSO) in two BRCA2-positive patients, a prophylactic contralateral mastectomy and BSO in one BRCA2-positive patient, and renal cell carcinoma surveillance and enrollment in a national Hereditary Leiomyomatosis and Renal Cell
Cancer (HLRCC) study in the patient with an $F H$ variant. While guidelines do not currently recommend a specific type of chemotherapy based on the presence or absence of deleterious germline variants, evidence suggests that tumors that arise in patients with deleterious germline BRCA1/BRCA2 variants may exhibit increased sensitivity to anthracyclines, platinum, and PARP inhibitors, and resistance to taxanes [11].

Our definition of actionable results was limited to deleterious and likely deleterious variants in genes with existing medical management guidelines. Several genes excluded from our return of results procedure may become actionable in the future as new guidelines are developed. If we used the definition of "potentially actionable" adopted by Kurian et al., which includes genes with a published association of two-fold or greater relative risk of breast cancer, the number of patients with variants in genes meeting criteria for return of results would increase from $13(9.7 \%)$ to $21(16.9 \%)$ [12]. The cancer risks associated with deleterious variants in these low-moderate penetrance genes are poorly defined, and it remains unclear whether additional surveillance or surgical management is warranted as the risk of contralateral breast cancer and other cancers is not well established. Thus, the clinical utility of returning such results to patients is uncertain.

The prevalence of deleterious germline variants identified and their potential clinical importance suggests that similar studies seeking to identify somatic variants for targeted cancer treatments should also prioritize the analysis of germline data. Classification of germline variants undoubtedly takes significant time and effort. When we tested filtering methods for tier one variants after the enrollment of 91 patients, filtering variants to include only those with a minor allele frequency of less than $1 \%$ that were either categorized as DM in HGMD or predicted to be protein truncating reduced the number of unique variants for review from 198 to 30, while capturing all deleterious variants. If the proposed filtering strategy had been used for all tier one and tier two variants for all 124 patients, the number of unique variants for review would have been reduced from 694 to 90, while capturing 27 of 28 deleterious variants-CHEK2 c.470T $>$ C (p.I157T) would have been missed as it is a missense variant that is listed as a "disease-associated polymorphism with additional supporting functional evidence," not DM, in HGMD. Dewey et al. recently reported that manual variant classification required a median of $54 \mathrm{~min}$ (range 5-223) per genetic variant [5]. Thus, we estimate that implementing these filters would have resulted in a time savings of approximately $544 \mathrm{~h}$ (almost 14 full work weeks), although sensitivity would have been reduced by excluding missense mutations that are not reported as DM in HGMD. ClinVar classification was not tested in our filtering techniques as 
the first full public release was not available at the time of study initiation, but was reviewed retrospectively. HGMD and ClinVar had nearly equal sensitivity, as 18/28 deleterious variants were classified as DM in HGMD and 19/28 deleterious variants were classified as pathogenic in ClinVar. Our data suggest that future studies could consider implementing germline analysis and return of results procedures using minor allele frequency, predicted protein impact, and inclusion in either HGMD or ClinVar as filters.

\section{Limitations}

This study has several limitations. CLIA-certified laboratory confirmation was not performed for all deleterious variants identified, and thus, for the non-clinically actionable variants, false positives may exist. It is possible that deleterious variants could have been missed after filtering strategies were implemented for tier two variants for the final 33 patients. Intronic, promoter, or other rare variants may have been missed given the limitations of whole exome sequencing. A large number of variants were classified as VUS, highlighting the current state of genomics knowledge and the difficulty of determining pathogenicity of variants. As demonstrated in previous studies, the burden of VUS generated by next-generation sequencing is a significant issue $[2,10,12]$. Because the median age of patients with breast cancer enrolled in our study was younger than the general population (51 vs. 61 years), the prevalence of deleterious variants is likely not generalizable to older breast cancer cohorts with low-risk tumor biology.

\section{Conclusion}

Deleterious germline variants in a variety of cancer susceptibility genes are frequent in breast cancer patients with high-risk tumor biology who were referred for neoadjuvant chemotherapy and their detection impacts medical management. Studies that seek to identify somatic variants using genomic sequencing technologies should also seek to identify actionable deleterious germline variants and return results to patients given the potential clinical implications.

Acknowledgments The BEAUTY study is funded in part by the Mayo Clinic Center for Individualized Medicine; Nadia's Gift Foundation; John P. Guider; the Eveleigh Family Career Development Award; and the Pharmacogenomics Research Network (PGRN). Other contributing groups include the Mayo Clinic Cancer Center and the Mayo Clinic Breast Specialized Program of Research Excellence (SPORE). We would like to thank Cloann Schultz for project management and support.
Declaration The experiments performed comply with the current laws of the United States of America.

\section{Compliance with ethical standards}

Conflict of interest The authors declare that they have no conflict of interest.

Research involving human rights All procedures performed in studies involving human participants were in accordance with the ethical standards of the institutional research committee and with the 1964 Helsinki declaration and its later amendments or comparable ethical standards. This article does not contain any studies with animals performed by any of the authors.

Informed consent Informed consent was obtained from all individual participants included in the study.

Open Access This article is distributed under the terms of the Creative Commons Attribution-NonCommercial 4.0 International License (http://creativecommons.org/licenses/by-nc/4.0/), which permits any noncommercial use, distribution, and reproduction in any medium, provided you give appropriate credit to the original author(s) and the source, provide a link to the Creative Commons license, and indicate if changes were made.

\section{References}

1. Abecasis GR, Auton A, Brooks LD, DePristo MA, Durbin RM, Handsaker RE, Kang HM, Marth GT, McVean GA (2012) An integrated map of genetic variation from 1,092 human genomes. Nature 491:56-65. doi:10.1038/nature11632

2. Bodian DL, McCutcheon JN, Kothiyal P, Huddleston KC, Iyer RK, Vockley JG, Niederhuber JE (2014) Germline variation in cancer-susceptibility genes in a healthy, ancestrally diverse cohort: implications for individual genome sequencing. PLoS One 9:e94554. doi:10.1371/journal.pone.0094554

3. Bombard Y, Robson M, Offit K (2013) Revealing the incidentalome when targeting the tumor genome. JAMA 310:795-796. doi:10.1001/jama.2013.276573

4. Castera L, Krieger S, Rousselin A, Legros A, Baumann JJ, Bruet O, Brault B, Fouillet R, Goardon N, Letac O, Baert-Desurmont S, Tinat J, Bera O, Dugast C, Berthet P, Polycarpe F, Layet V, Hardouin A, Frebourg T, Vaur D (2014) Next-generation sequencing for the diagnosis of hereditary breast and ovarian cancer using genomic capture targeting multiple candidate genes. Eur J Hum Genet. doi:10.1038/ejhg.2014.16

5. Dewey FE, Grove ME, Pan C, Goldstein BA, Bernstein JA, Chaib H, Merker JD, Goldfeder RL, Enns GM, David SP, Pakdaman N, Ormond KE, Caleshu C, Kingham K, Klein TE, Whirl-Carrillo M, Sakamoto K, Wheeler MT, Butte AJ, Ford JM, Boxer L, Ioannidis JP, Yeung AC, Altman RB, Assimes TL, Snyder M, Ashley EA, Quertermous T (2014) Clinical interpretation and implications of whole-genome sequencing. JAMA 311:1035-1045. doi:10.1001/ jama.2014.1717

6. Ding SL, Yu JC, Chen ST, Hsu GC, Kuo SJ, Lin YH, Wu PE, Shen CY (2009) Genetic variants of BLM interact with RAD51 to increase breast cancer susceptibility. Carcinogenesis 30:43-49. doi:10.1093/carcin/bgn233

7. Dorschner MO, Amendola LM, Turner EH, Robertson PD, Shirts BH, Gallego CJ, Bennett RL, Jones KL, Tokita MJ, Bennett JT, Kim JH, Rosenthal EA, Kim DS, Tabor HK, Bamshad MJ, 
Motulsky AG, Scott CR, Pritchard CC, Walsh T, Burke W, Raskind WH, Byers P, Hisama FM, Nickerson DA, Jarvik GP (2013) Actionable, pathogenic incidental findings in 1,000 participants' exomes. Am J Hum Genet 93:631-640. doi:10.1016/j. ajhg.2013.08.006

8. Exome Variant Server In: NHLBI GO Exome Sequencing Project (ESP)

9. Gracia-Aznarez FJ, Fernandez V, Pita G, Peterlongo P, Dominguez O, de la Hoya M, Duran M, Osorio A, Moreno L, Gonzalez-Neira A, Rosa-Rosa JM, Sinilnikova O, Mazoyer S, Hopper J, Lazaro C, Southey M, Odefrey F, Manoukian S, Catucci I, Caldes T, Lynch HT, Hilbers FS, van Asperen CJ, Vasen HF, Goldgar D, Radice P, Devilee P, Benitez J (2013) Whole exome sequencing suggests much of non-BRCA1/BRCA2 familial breast cancer is due to moderate and low penetrance susceptibility alleles. PLoS One 8:e55681. doi:10.1371/journal.pone. 0055681

10. Johnston JJ, Rubinstein WS, Facio FM, Ng D, Singh LN, Teer JK, Mullikin JC, Biesecker LG (2012) Secondary variants in individuals undergoing exome sequencing: screening of 572 individuals identifies high-penetrance mutations in cancer-susceptibility genes. Am J Hum Genet 91:97-108. doi:10.1016/j. ajhg.2012.05.021

11. Kriege M, Jager A, Hooning MJ, Huijskens E, Blom J, van Deurzen CH, Bontenbal M, Collee JM, Menke-Pluijmers MB, Martens JW, Seynaeve C (2012) The efficacy of taxane chemotherapy for metastatic breast cancer in BRCA1 and BRCA2 mutation carriers. Cancer 118:899-907. doi:10.1002/ cncr.26351

12. Kurian AW, Hare EE, Mills MA, Kingham KE, McPherson L, Whittemore AS, McGuire V, Ladabaum U, Kobayashi Y, Lincoln SE, Cargill M, Ford JM (2014) Clinical evaluation of a multiplegene sequencing panel for hereditary cancer risk assessment. J Clin Oncol 32:2001-2009. doi:10.1200/JCO.2013.53.6607

13. Landrum MJ, Lee JM, Riley GR, Jang W, Rubinstein WS, Church DM, Maglott DR (2014) ClinVar: public archive of relationships among sequence variation and human phenotype. Nucleic Acids Res 42:D980-985. doi:10.1093/nar/gkt1113

14. Lehtonen HJ, Kiuru M, Ylisaukko-Oja SK, Salovaara R, Herva R, Koivisto PA, Vierimaa O, Aittomaki K, Pukkala E, Launonen V, Aaltonen LA (2006) Increased risk of cancer in patients with fumarate hydratase germline mutation. J Med Genet 43:523-526. doi:10.1136/jmg.2005.036400

15. Lindor NM, McMaster ML, Lindor CJ, Greene MH (2008) Concise handbook of familial cancer susceptibility syndromessecond edition. J Natl Cancer Inst Monogr 38:1-93. doi:10.1093/ jncimonographs/lgn001

16. Mwenifumbo JC, Marra MA (2013) Cancer genome-sequencing study design. Nat Rev Genet 14:321-332. doi:10.1038/nrg3445

17. Online Mendelian Inheritance in Man $\left(\mathrm{OMIM}^{\circledR}\right)$ McKusickNathans Institute of Genetic Medicine, Johns Hopkins University, Baltimore
18. Pasche B, Absher D (2011) Whole-genome sequencing: a step closer to personalized medicine. JAMA 305:1596-1597. doi:10. 1001/jama.2011.484

19. Prokofyeva D, Bogdanova N, Dubrowinskaja N, Bermisheva M, Takhirova Z, Antonenkova N, Turmanov N, Datsyuk I, Gantsev S, Christiansen H, Park-Simon TW, Hillemanns P, Khusnutdinova E, Dork T (2013) Nonsense mutation p. Q548X in BLM, the gene mutated in Bloom's syndrome, is associated with breast cancer in Slavic populations. Breast Cancer Res Treat 137:533-539. doi:10.1007/s10549-012-2357-1

20. Rennert G, Lejbkowicz F, Cohen I, Pinchev M, Rennert HS, Barnett-Griness O (2012) MutYH mutation carriers have increased breast cancer risk. Cancer 118:1989-1993. doi:10. $1002 /$ cncr.26506

21. Richards CS, Bale S, Bellissimo DB, Das S, Grody WW, Hegde MR, Lyon E, Ward BE (2008) ACMG recommendations for standards for interpretation and reporting of sequence variations: Revisions 2007. Genet Med 10:294-300. doi:10.1097/GIM 0b013e31816b5cae

22. Sherry ST, Ward MH, Kholodov M, Baker J, Phan L, Smigielski EM, Sirotkin K (2001) dbSNP: the NCBI database of genetic variation. Nucleic Acids Res 29:308-311

23. Solyom S, Winqvist R, Nikkila J, Rapakko K, Hirvikoski P, Kokkonen H, Pylkas K (2011) Screening for large genomic rearrangements in the FANCA gene reveals extensive deletion in a Finnish breast cancer family. Cancer Lett 302:113-118. doi:10. 1016/j.canlet.2010.12.020

24. Stadler ZK, Schrader KA, Vijai J, Robson ME, Offit K (2014) Cancer genomics and inherited risk. J Clin Oncol 32:687-698. doi:10.1200/JCO.2013.49.7271

25. Stenson PD, Mort M, Ball EV, Howells K, Phillips AD, Thomas NS, Cooper DN (2009) The Human Gene Mutation Database: 2008 update. Genome Med 1:13. doi:10.1186/gm13

26. Szabo C, Masiello A, Ryan JF, Brody LC (2000) The breast cancer information core: database design, structure, and scope. Hum Mutat 16:123-131. doi:10.1002/1098-1004(200008)

27. Thompson ER, Doyle MA, Ryland GL, Rowley SM, Choong DY, Tothill RW, Thorne H, Barnes DR, Li J, Ellul J, Philip GK, Antill YC, James PA, Trainer AH, Mitchell G, Campbell IG (2012) Exome sequencing identifies rare deleterious mutations in DNA repair genes FANCC and BLM as potential breast cancer susceptibility alleles. PLoS Genet 8:e1002894. doi:10.1371/journal. pgen.1002894

28. Wang Z, Xu Y, Tang J, Ma H, Qin J, Lu C, Wang X, Hu Z, Shen $\mathrm{H}$ (2009) A polymorphism in Werner syndrome gene is associated with breast cancer susceptibility in Chinese women. Breast Cancer Res Treat 118:169-175. doi:10.1007/s10549-009-0327-Z 